\section{Michigan Technological

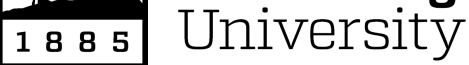

Michigan Technological University Digital Commons @ Michigan Tech

8-15-2012

\title{
Open Source Research in Sustainability
}

Joshua M. Pearce

Michigan Technological University

Follow this and additional works at: https://digitalcommons.mtu.edu/materials_fp

\section{Recommended Citation}

oshua M. Pearce, "Open Source Research in Sustainability", Sustainability the Journal of Record, 5(4), pp. 238-243, 2012. http://digitalcommons.mtu.edu/materials_fp/44 
Published as: Joshua M. Pearce, “Open Source Research in Sustainability”, Sustainability the Journal of Record, 5(4), pp. 238-243, 2012. DOI:

http://dx.doi.org/10.1089/sus.2012.9944

\author{
Open Source Research in Sustainability \\ Joshua M. Pearce \\ Department of Materials Science \& Engineering \\ Department of Electrical \& Computer Engineering \\ Michigan Technological University \\ 601 M\&M Building \\ 1400 Townsend Drive \\ Houghton, MI 49931-1295 \\ 906-487-1466 \\ pearce@mtu.edu
}

\begin{abstract}
Most academics, who as researchers and teachers dedicate their lives to information sharing, would likely agree with much of the hacker ethic, which is a belief that information-sharing is a powerful positive good and there is an ethical duty to share expertise by writing free and open-source code and facilitating access to information wherever possible. However, despite a well-established gift culture similar to that of the open source software movement in academic publishing and the tenure process many academics fail to openly provide the "source” (e.g. data sets, literature reviews, detailed experimental methodologies, designs, and open access to results) of their research. Closed research is particularly egregious when it could be used to accelerate the transition to a sustainable world and this transition is hobbled by antiquated research methodologies that slow the diffusion of innovation. To overcome this challenge, this paper reports on several experiments to embrace the use of open source methodologies in research for applied sustainability. Open-access and open-edit Internet technologies were used as both real-time research tools and a means of disseminating findings on appropriate technology and sustainability-related research to the broadest possible audience. The results of experiments on open source research found that more rapid deployment of sustainability technologies is possible by building on the foundations of the hacker ethic with i) massive peer-review in the development of background material and experimental design, ii) increased visibility, which leads to iii) increased funding opportunities and improved student recruitment, and iv) improved student research-related training and education.
\end{abstract}

\title{
Introduction
}

The vast majority of academic research is not focused on problems related to sustainability and surprisingly, even much of it that is, is effectively removed from deployment by intellectual property (IP) law (e.g. patents and copyrights). ${ }^{1}$ The results of this restricted and closed model of technological development and deployment contribute to environmental desecration and the current global ecological decline, widespread poverty and social injustice seen around the globe, which is directly responsible for a morally and ethically unacceptable level of human suffering and death. ${ }^{1}$ This waste of human life could be prevented by known (to humanity as a whole) technologies, many of which are simply not available to those who need it. Availability is restricted by both the cost of access (such as pay-to-view 
Published as: Joshua M. Pearce, “Open Source Research in Sustainability”, Sustainability the Journal of Record, 5(4), pp. 238-243, 2012. DOI: http://dx.doi.org/10.1089/sus.2012.9944

articles on sustainability or sustainable technologies under copyright ${ }^{\mathrm{i}}$ and by companies wielding patent law to maximize profit above all other costs (e.g. restricting the sale of antiretroviral drugs to treat HIV in Africa). ${ }^{2}$ Hackers offer an intriguing pathway to a solution to these problems for academics and this paper explores that pathway.

Contrary to popular misrepresentations in the media, the original and real definition, of a hacker, is a computer programmer who shares an anti-authoritarian approach to software development now associated with free and open source software (FOSS). ${ }^{3}$ FOSS is software that is freely licensed to grant users the right to use, copy, study, change, and improve its design through the availability of its source code or be "open source”. The open source movement emerged as a fundamentally new, decentralized $^{4}$, participatory and transparent system to develop software in contrast to the closed box, industrial, top-down, and proprietary approach. ${ }^{5}$ The open source paradigm also usually includes viral components that require users to share improvements with the community under the same open/free terms of the original work. ${ }^{6}$ Through these principles of sharing and open-access, open source treats users as developers by encouraging contribution, recognizing good work through peer approval, and propagating superior code. ${ }^{7}$ This philosophy of the open source movement is described by Levy as the 'hacker ethic' with the following general principles: i) sharing, ii) openness, iii) decentralization, iv) free access, and v) world improvement. ${ }^{4}$ This philosophy is enabled by the gift culture of open source, in which recognition of an individual is determined by the amount of knowledge given away. ${ }^{8}$ This type of philosophy should be familiar to many in academia, which has also historically followed a gift culture, which rewards contributors through a process of peer review.

Most academics, who as researchers and teachers, dedicate their lives to information sharing, ${ }^{\text {ii }}$ would likely agree with the majority of the hacker ethic, but despite a well-established gift culture in the tenure process many academics fail to open source their research (e.g. share components of research such as literature reviews for preparation, data sets, detailed experimental methodologies or protocols, experimental and equipment designs, and open access to results). This is perhaps most problematic when this research could be used to accelerate the transition to a sustainable world and thus, this transition is handicapped by antiquated research methodologies that slow the diffusion of innovation. Open source methodologies, however, can be applied to sustainability research to overcome this handicap to develop open source appropriate technology (OSAT), which are environmentally, socially, technically, culturally, and economically appropriate for a community. ${ }^{9}$ The use of OSAT has enormous potential to assist in sustainable development because it collaboratively organizes information, project examples, and best practices ${ }^{10}$ and is set to expand rapidly as organizations utilize its information transfer and collaboration capabilities. ${ }^{11}$ Following the open source paradigm, anyone can both learn how to make and use AT free of IP concerns and add to the collective open source knowledge ecosystem or "knowledge commons" by contributing ideas, designs, observations, experimental data, deployment logs, etc. The free nature of the knowledge also provides lower costs, particularly for those technologies that do not benefit to a large degree from scale of manufacture (e.g. open source hardware such as 3D printers for both the design, but also the production of OSAT). ${ }^{12,13}$

This study utilized open-access and open-edit Internet technologies as both real-time research tools and a means of disseminating findings on appropriate technology and sustainability-related

i Even proceedings from a conference on "Science and Technology for Humanity" with articles on open source appropriate technology for sustainable development such as reference 1 cost US\$30 for non-subscribers.

ii Open source sharing of educational materials (e.g. class handouts, lecture notes, homework problems, projects, and Libre Office/Open Office/PowerPoint slides) is already well established. For example, the Center for Sustainable Engineering hosts an open source website for education materials related to sustainability at http://www.csengin.org . 
Published as: Joshua M. Pearce, “Open Source Research in Sustainability”, Sustainability the Journal of Record, 5(4), pp. 238-243, 2012. DOI: http://dx.doi.org/10.1089/sus.2012.9944

research to the broadest possible audience. While legally navigating copyright restrictions the following were shared in addition to open access allowances for the resultant papers: literature reviews, protocols, result summaries and both static and live data. The results of these open source research experiments are discussed and conclusions are drawn to provide more rapid deployment of sustainability technologies by building on the foundations of the hacker ethic.

\section{Methodology}

\section{How to Use Appropedia to Enhance Sustainability Research}

In order to test the use of open source methodologies in research for applied sustainability the website Appropedia.org was used for this study over 4 years. The Appropedia Foundation hosts this primary site for collaborative solutions in sustainability, poverty reduction and international development on the Internet (Figure 1). Appropedia is an advertising-free Mediawiki-based website like Wikipedia, where a large number of participants can create and modify the content directly from their web browsers. In this study, the open source research was applied to sustainability-focused research in three ways: 1) literature reviews, 2) protocols, and 3) summarizing the results of research and providing data for greater dissemination. In all cases, Appropedia pages are held in a category for the research group for credit and placed in appropriate content categories (e.g. Category:Photovoltaics). All of the members of the Queen's Applied Sustainability (QAS) and the Michigan Tech Open Sustainability Technology (MOST) research groups were required to publish the following on Appropedia:

\section{Literature Reviews}

The guidelines used to create a literature review page ${ }^{\mathrm{iii}}$ for a research topic include:

- Maintain records of all searches (e.g. Google Scholar for "Solar energy").

- Make a citation list for all the articles found relevant to the topic and arrange chronologically.

- Use the open source web-browser Firefox ${ }^{\mathrm{iv}}$ with the open source extension Zotero ${ }^{\mathrm{v}}$ to organize articles. Include full citation exported from Zotero in the format of the target journal.

- Hyperlink the citation to the available electronic file (DOI) and if possible include a link to an open access version.

\section{Protocols}

In this study experimental protocols and methods were shared in complete detail on Appropedia so that others may use, replicate, and improve upon the methods. The protocols were meant to be:

- Clear enough to allow anyone to replicate the work, particularly future students.

- Detailed enough to allow text of the experimental apparatus and general protocols to be used directly in articles.

- Transparent enough for others to replicate and scale throughout the rest of the world by including details of software that are linked to the sources on the web and strategies to use

iii See for example: http://www.appropedia.org/Category:MOST literature reviews

iv http://www.mozilla.org/en-US/

v http://www.zotero.org/ 
Published as: Joshua M. Pearce, “Open Source Research in Sustainability”, Sustainability the Journal of Record, 5(4), pp. 238-243, 2012. DOI:

http://dx.doi.org/10.1089/sus.2012.9944

FOSS and develop open source hardware for others.

\section{Summarizing Results and Outreach}

Finally when the research project is completed and the article has been accepted for publication the members of the group are required to summarize their work in a completed project page providing:

- A summary of the project and key findings.

- Links to related literature reviews, protocols, and sources of raw data (both real time and static).

- The full citation for the article with a link to the article at the journal via DOI and an open access version of the published paper.

- Enhanced figures (e.g. color figures), embedded YouTube videos on how to do things or a brief summary making the work more accessible to the layperson.

- Internal hyperlinks to all keywords within Appropedia.

- In some cases the projects received an Appropedia status tag as seen in Figure 2.

\section{Results and Discussion}

\section{Advantages of Open Access and Open Source Research for the Academic}

Overall the experiments realizing the hacker ethic for open source research into applied sustainability were successful as determined by an increase in quality and quantity of both research and deployment. This success was driven by both the web2.0 platform and by simply providing open access to much of the research. First, there were several advantages for the academic that were observed using Appropedia for open source research related to it being an open wiki. Wikis are easy to edit with a relatively shallow learning curve (e.g. a typical student can master the basics of wiki markup in under 30 minutes). This allowed new students to quickly begin making real contributions to the effort of the group. Multiple members of the research team were able to edit and thus improve a page (e.g. a literature search or methodology) at the same time or from different locations. Utilizing the wiki keeps track of the status of an individual or teams of researchers and enables updates to literature reviews from prior years. It also easily enables multiple members of the group to collaborate asynchronously. The collaborative advantages to this were very useful as researchers could work from home and thus reduce commuting time during certain aspects of the research while still contributing. Most remarkably, it facilitates others outside of the research group to assist. Literature reviews, in particular, were observed to be more complete, accurate and up-to-date because of external assistance. Other examples of assistance from Appropedia users not affiliated with the group encountered during this study included: i) giving helpful comments on the discussion tab of group pages, ii) making grammar/spelling corrections, and iii) adding categories and hyperlinking either to or within work that a group has made, which adds to the value of the work.

Improvements were also observed in methods. The influence of corporate thought on universities is well established ${ }^{14}$ and today at many universities there is pressure to avoid having IP 'scooped' by patenting and or commercializing research to prevent replication without the academic's employer benefiting financially. ${ }^{15}$ This corporate view of research has now infiltrated the academic literature. As can be seen in many fields, normally the experimental section of journal articles is the shortest and most opaque section of a manuscript and potentially "threaten the foundation of scientific 
Published as: Joshua M. Pearce, “Open Source Research in Sustainability”, Sustainability the Journal of Record, 5(4), pp. 238-243, 2012. DOI: http://dx.doi.org/10.1089/sus.2012.9944

discourse”16 and even these details are not sometimes released until provisional patents have been filed. In addition, most experimental protocols that are standards are not open access and many require substantial fees to even view. Being shared online as a quasi-publication ensured quality in student experimental and simulation design, while making the work of the groups easy to replicate.

Appropedia is currently the largest wiki dedicated to appropriate technology and sustainability, which gave the group research widespread readership and accessibility. Using a page view counting toolvi it was found that group work had been accessed over 500,000 and 200,000 times over 3 years for QAS and less than one year for MOST, respectively as seen in Table 1.

Table 1. Page views from open source research on May 9, 2012.

\begin{tabular}{|c|c|c|}
\hline Category Type & QAS & MOST \\
\hline Initiation Date & Spring 2009 & Fall 2011 \\
\hline & \multicolumn{2}{|c|}{ Page Views $^{a}$} \\
\hline Main Category & 182,000 & 118,000 \\
\hline Literature Reviews & 176,000 & 71,700 \\
\hline Methods and Protocols & 104,000 & 8,300 \\
\hline Completed Projects & 43,000 & 16,900 \\
\hline Totals & 505,000 & 215,000 \\
\hline
\end{tabular}

${ }^{\text {a }}$ Data overlap exists between groups due to collaboration.

This exposure can directly lead to funding opportunities. An example of this includes one of the research projects highlighted as part of this open research experiment on the effects of snow on solar photovoltaic performance. Due to Appropedia posting the details of the experiment as it was underway, the project page received the top rank in Google searches. This exposure resulted in a successful open source partnership being formed between 20 organizations all of which directly contributed funding, equipment and in-kind support to produce the Open Solar Outdoors Test Field (OSOTF), which provides critical data and research in the public domain for solar PV systems optimization in snowy environments. ${ }^{17}$ Unlike many other projects, the OSOTF is organized under open source principles guided by the hacker ethic. All data and analysis when completed is made freely available to the entire photovoltaic community and the general public and already much of the data including real time photos of the OSOTF are available in five minute increments live. ${ }^{\text {vii }}$ The collaborating partners in the OSOTF project were willing to overcome the challenges of doing open source research because they saw value in having free access to critical research data that would be useful for product improvement, more reliable predictions of performance for funding, and reductions in solar electric system losses. It is clear from the number of collaborators who contacted the group that many of the partners would never have known about the project without the group using the open source research approach. In addition the coverage assisted in attracting graduate student researchers.

Thus, the advantages to providing some open access to work underway for any researcher wanting to disseminate their research is clear. Putting summaries of work on Appropedia with DOI links to the original article and ideally to an open access version will help increase the number of

vi http://pequals.com/appro count.php

vii http://snowstudy.ati.sl.on.ca/ 
Published as: Joshua M. Pearce, “Open Source Research in Sustainability”, Sustainability the Journal of Record, 5(4), pp. 238-243, 2012. DOI: http://dx.doi.org/10.1089/sus.2012.9944

people reading a given article. It has been postulated that if more people read it then open access will also raise the citation rate and h-index. ${ }^{18}$ Although, such claims are under debate in the literature, this group's experience particularly with citations from non-North American researchers provides some modest evidence for the effect. This effect may provide a critical advantage in the tenure process for academics providing 'aggressive open access' depending on the university.

There are also advantages to using an open research approach within a research group. First, the open literature reviews are extremely effective for passing on knowledge to the next generation of students, finding references read when writing a paper, and keeping track of background research. Open protocols are important for maintaining standards within the group research - but also building more easily on the work of others. In some disciplines the sharing of free and open source protocols is very advanced (e.g. OpenWetWare.org, which is an effort to promote the sharing of information in biology and biological engineering), while other disciplines (e.g. mechanical engineering relying on proprietary ASME standards) are not. There are several generic open source research websites now that can provide the needs for nearly any discipline such as openresearch.org (a semantic wiki for the sciences) or myexperiment.org (which publishes scientific workflows).

There are thus advantages of greater adoption of open access and open source research for students. Free (zero cost) access to the latest literature is an intuitively obvious advantage, which should result in better-quality lab reports, term papers, and student writing. However, students also benefit from faster learning and higher-quality information from the aggressive and potentially massive peer-review made possible by open research. Finally, there is clear evidence that combining open research with service learning in the classroom provides superior learning outcomes for students. ${ }^{19}$

\section{Challenges and Discussion}

To publish in the peer reviewed literature, unfortunately (and perhaps for not much longer as IP in the form of patent and copyright law are being challenged ${ }^{20}$ ) authors normally must sign away IP rights (copyright). Thus, pre-publishing work on the Internet before submission to the journal is a concern. Until IP rules are revised or completely eliminated, this risk can be avoided by limiting the type of information that is shared prior to publication. In addition, most publishers now allow the posting of pre-prints in institutional repositories after submission (e.g. as does the physics community in arXiv.org).

Vandalism is a concern on open wikis. During the course of these experiments with open research, several cases of vandalism were observed, but were easily and immediately corrected by Appropedia administrators as all work in a page is tracked its history (Figure 3). Users can add pages to their watchlists and be can be notified if it is edited by someone else and request administrators to "lock" pages to avoid further edits on request (comments are still possible on the discussion tab). During the course of this experiment over 4 years these additional levels of security were found to be unnecessary. All major articles were published in the traditional peer-reviewed literature and made available via open access in institutional repositories. All of the other components were left as open edit to enable future users to improve upon them.

More scientists using this approach would likely improve the scale of the benefits. In a vibrant and well-populated open sustainability research community maintaining literature reviews would become a community affair and not the primary work of a single group. If the majority of researchers simply added their own work this type of initiative would be significantly improved. Similarly in many fields custom equipment designs, methods, and software are kept private to an institution or research 
group - if these were provided openly in some form of website, via a wiki protocol or 'instructable' the benefits for all in the particular field would be enhanced appreciably.

These results indicate that sustainability research can be accelerated and the results disseminated faster if an open source research methodology is used. In the work that this journal focuses on - encouraging sustainability, ethical considerations must also play a role. Is it ethical not to provide sustainability research information for free to others if so doing would be more likely to create a sustainable society?

\section{Conclusions}

This paper reported on several experiments to embrace the use of open source methodologies in research for applied sustainability. It utilized open-access publication and the open-edit appropedia.org platform as both a real-time research tool and a means of disseminating findings on appropriate technology and sustainability-related research to the broadest possible audience. This approach was found to be useful for sharing several aspects of research including the literature preparations, methods, result summaries and data. The results of experiments on open source research found that more rapid deployment of applied sustainability technologies is possible following the hacker ethic by profiting from i) massive peer-review in the development of background material and experimental design, ii) increased visibility and public relations, which leads to iii) increased funding opportunities and improved student recruitment, and iv) improved student research-related training and education.

\section{Acknowledgments}

The author would like the acknowledge the Appropedia Foundation for providing the platform to do this research. An earlier version of this paper was presented as "What Hackers Can Teach Academics: Open Source Research in Applied Sustainability", at the $9^{\text {th }}$ Ball State University Greening of the Campus Conference, Muncie Indiana, 18-21 March, 2012.

\section{Author Disclosure Statement}

No competing financial interests exist. 
Published as: Joshua M. Pearce, “Open Source Research in Sustainability”, Sustainability the Journal of Record, 5(4), pp. 238-243, 2012. DOI: http://dx.doi.org/10.1089/sus.2012.9944

\section{Figures}

Figure 1. Appropedia is for collaborative solutions in sustainability, appropriate technology and poverty reduction.

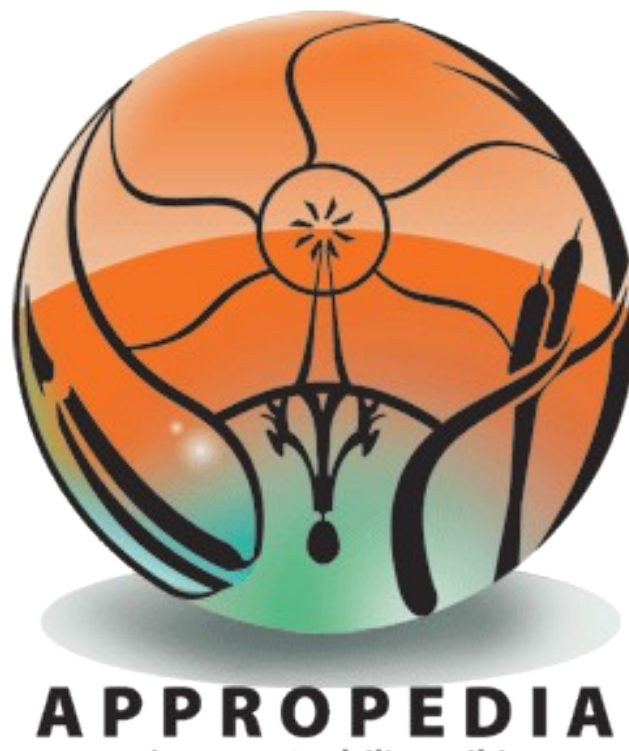

the sustainability wiki.

Figure 2. Appropedia status tags. The status of a particular OSAT can be shown easily by adding the wiki markup templates to the top of an AT project page. The coloring scheme denotes the hierarchy of risk: with OSATs that have only been designed in red, those modeled in orange, prototyped in yellow, verified in light green and deployed in dark green.

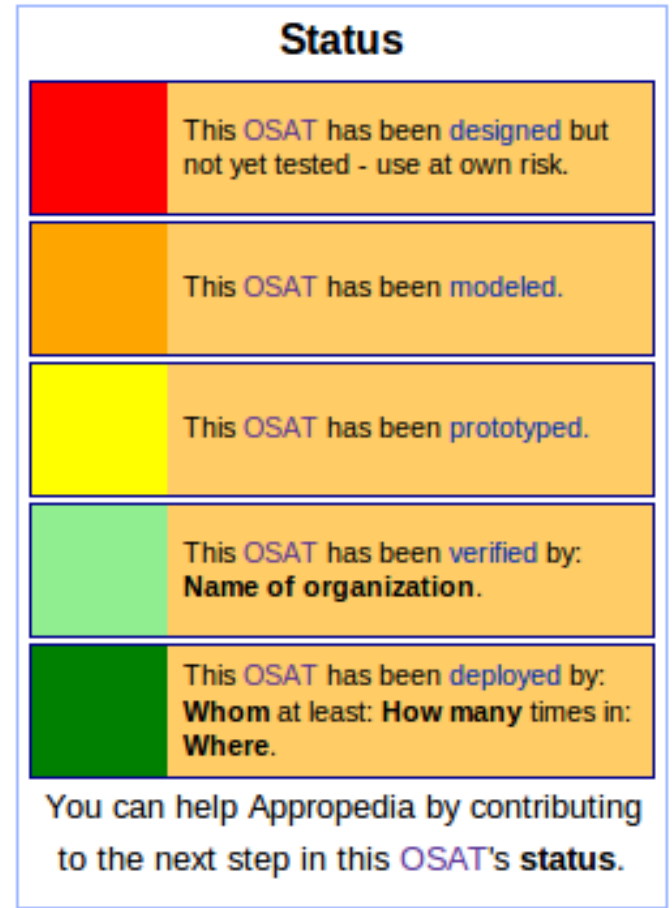


Published as: Joshua M. Pearce, “Open Source Research in Sustainability”, Sustainability the Journal of Record, 5(4), pp. 238-243, 2012. DOI: http://dx.doi.org/10.1089/sus.2012.9944

\begin{tabular}{|c|c|c|c|c|c|c|c|c|}
\hline page & discussion & edit & history & & & & & \\
\hline \multirow{2}{*}{\multicolumn{3}{|c|}{ Revision history of }} & \multicolumn{6}{|c|}{ Appropriate technology $\cdot$ Construction $\cdot$ Food \& Agriculture $\cdot$ Green living $\cdot$ Energy $\cdot$ Solar $\cdot$ Health \& Safety $\cdot$ Medical Devices $\cdot$ Projects $\cdot$ Transport $\cdot$ Water } \\
\hline & & & "Greenhouses li & literature r & eview" & $+1\}$ & ๔3 Like & E Be the first of your friends to like this. \\
\hline \multicolumn{9}{|c|}{ View logs for this page } \\
\hline \multicolumn{9}{|c|}{ — Browse history } \\
\hline \multicolumn{3}{|c|}{ From year (and earlier): } & From month (and earlier): & all & $\square$ Deleted only & Go & & \\
\hline
\end{tabular}

The following are previous versions of Greenhouses literature review.

To see the difference between two versions, check their radio buttons and click Compare selected versions.
Compare selected revisions
- (Difference to current $\mid$...to last)
- (Difference to current $\mid$...to last)
03:29, 17 November 2009130.15 .116 .247 (Talk | Contributions) (11,371 bytes) ( $\rightarrow$ Greenhouse waste heat) (undo)
(Difference to current | ...to last)
18:52, 6 November 2009 R.Andrews (Talk | Contributions) (9,881 bytes) ( $\rightarrow$ Greenhouse waste heat) (undo)
- (Difference to current $\mid$...to last)
18:51, 6 November 2009 R.Andrews (Talk | Contributions) (9,815 bytes) ( $\rightarrow$ Greenhouse waste heat) (undo)
- (Difference to current | ...to last)
15:43, 5 November 2009 R.Andrews (Talk | Contributions) (9,171 bytes) ( $\rightarrow$ Greenhouse waste heat) (undo)
- (Difference to current | ...to last)
14:37, 5 November 2009 R.Andrews (Talk | Contributions) (8,065 bytes) ( $\rightarrow$ Greenhouse waste heat) (undo)
- (Difference to current | ...to last)
01:44, 3 November 2009 Frieddog (Talk | Contributions) (7,491 bytes) ( $\rightarrow$ CO2 enrichment) (undo)
- (Difference to current $\mid$....to last)
01:30, 3 November 2009 Frieddog (Talk | Contributions) (7,518 bytes) ( $\rightarrow$ Greenhouse waste heat) (undo)
- (Difference to current $\mid$...to last)
01:05, 3 November 2009 Frieddog (Talk | Contributions) (7,519 bytes) ( $\rightarrow$ Greenhouses background) (undo)
- (Difference to current $\mid$...to last)
22:13, 2 November 2009 R.Andrews (Talk | Contributions) (7,500 bytes) ( $\rightarrow$ Greenhouses background) (undo)
22:08, 2 November 2009 R.Andrews (Talk | Contributions) (7,455 bytes) ( $\rightarrow$ Greenhouses background) (undo)
- (Difference to current $\mid$....to last)
22:08, 2 November 2009 R.Andrews (Talk | Contributions) (7,444 bytes) ( $\rightarrow$ Greenhouses background) (undo)
- (Difference to current $\mid$...to last)
21:21, 2 November 2009 R.Andrews (Talk | Contributions) (6,430 bytes) ( $\rightarrow$ CO2 enrichment) (undo)
- (Difference to current $\mid$....to last)
21:20, 2 November 2009 R.Andrews (Talk | Contributions) (6,396 bytes) ( $\rightarrow$ Greenhouse waste heat) (undo)

Figure 3. Example of a history tab in Appropedia. All mistaken edits or vandalism can be turned back immediately by clicking the undo hyperlink. This page also shows a record of assistance from outside the group as the user Frieddog was not a group member. 


\section{References}

1 Pearce JM. Mushtaq U. Overcoming Technical Constraints for Obtaining Sustainable Development with Open Source Appropriate Technology. Proceedings of the Science and Technology for Humanity (TIC-STH), 2009 IEEE Toronto International Conference 2009; 814-820.

2 Shantharam Y. The cost of life: patent laws, the WTO, and the HIV/AIDS pandemic. Undercurrent 2005; 2(2): 48-56.

3 Levy S. Hackers: Heroes of the Computer Revolution. Doubleday, New York,1984.

4 Raymond ES. The cathedral and the bazaar. First Monday 1998; 3(3).

5 Mockus A. Fielding RT. Herbsleb JD. Two cases studies of open source software development: Apache and Mozilla. ACM Transactions on Software Engineering and Methodology 2002;11(3): 309-346.

6 Hansen M. Kohntopp K. Pfitzmann A. The open source approach - opportunities and limitations with respect to security and privacy. Computers \& Security 2002; 21(5): 461-471.

7 Weber S. The political economy of open source software. Berkeley Roundtable on the International Economy, Working Paper 140, 2000.

8 Bergquist M. Ljungberg J. The power of gifts: organizing social relationships in open source communities, Information Systems Journal 2001; 11: 305-320.

9 Buitenhuis AJ. Zelenika I. Pearce JM. Open Design-Based Strategies to Enhance Appropriate Technology Development. Proceedings of the 14th Annual National Collegiate Inventors and Innovators Alliance Conference : Open 2010;1-12.

10 Pearce JM. The Case for Open Source Appropriate Technology. Environment, Development and Sustainability 2012; 14:425-431.

11 Zelenika I. Pearce JM. Barriers to Appropriate Technology Growth in Sustainable Development. Journal of Sustainable Development 2011; 4(6): 12-22.

12 Pearce JM. Morris Blair C. Laciak KJ. Andrews R. Nosrat A. Zelenika-Zovko I. 3-D Printing of Open Source Appropriate Technologies for Self-Directed Sustainable Development. Journal of Sustainable Development 2010; 3(4): 17-29.

13 Symes MD. Kitson PJ. Yan J., Richmond C.J., Cooper GJT. Bowman, RW. Vilbrandt T. Cronin L. Integrated 3D-printed reactionware for chemical synthesis and analysis. Nature Chemistry 2012; 4: 349-354.

14 Chan AS., \& Fisher D. Exchange university: Corporatization of academic culture. University of British Columbia Press, Vancouver, 2008.

15 Lieberwitz RL. Educational Law: The Corporatization of Academic Research: Whose Interests Are Served?, 38 AKRON L. REV. 2005; 759: 764-65.

16 Gelman IJ. Opinion: Missing Methods. The Scientist 2012. Available http://the-scientist.com/2012/05/03/opinionmissing-methods/ (Last accessed on [May 9, 2012]).

17 Pearce JM. Babasola A. Andrews R. Open Solar Photovoltaic Systems Optimization. Proceedings of the 16th Annual National Collegiate Inventors and Innovators Alliance Conference, 2012; 1-7.

18 Harnad S. Brody T. Comparing the impact of Open Access (OA) vs. non-OA articles in the same journals. D-Lib Magazine 2004; 10.

19 Pearce JM. Appropedia as a Tool for Service Learning in Sustainable Development. Journal of Education for Sustainable Development 2009;3(1): 47-55.

20 Kinsella NS. Against Intellectual Property, Journal of Libertarian Studies 2001;15(2): 1-53. 